Resubmitted to Appl. Surf. Sci., September 2015

\title{
SYNCHRONISED ELECTRICAL MONITORING AND HIGH SPEED VIDEO OF BUBBLE GROWTH ASSOCIATED WITH INDIVIDUAL DISCHARGES DURing Plasma Electrolytic OXIDATion
}

\author{
SC Troughton ${ }^{1}$, A Nominé $^{2}$, AV Nominé ${ }^{2}$, Henrion $^{3} \&$ TW Clyne ${ }^{1 *}$ \\ ${ }^{1}$ Department of Materials Science \& Metallurgy \\ Cambridge University \\ 27 Charles Babbage Road, Cambridge CB3 0FS, UK \\ ${ }^{2}$ Department of Physical Sciences \\ Open University \\ Walton Hall, Milton Keynes MK7 6AA, UK \\ ${ }^{3}$ Institut Jean Lamour \\ CNRS, Université de Lorraine \\ 54011 Nancy, France
}

\begin{abstract}
Synchronised electrical current and high speed video information are presented from individual discharges on Al substrates during PEO processing. Exposure time was $8 \mu$ s and linear spatial resolution $9 \mu \mathrm{m}$. Image sequences were captured for periods of $2 \mathrm{~s}$, during which the sample surface was illuminated with short duration flashes (revealing bubbles formed where the discharge reached the surface of the coating). Correlations were thus established between discharge current, light emission from the discharge channel and (externally-illuminated) dimensions of the bubble as it expanded and contracted. Bubbles reached radii of $500 \mu \mathrm{m}$, within periods of $100 \mu \mathrm{s}$, with peak growth velocity about $10 \mathrm{~m} / \mathrm{s}$. It is deduced that bubble growth occurs as a consequence of the progressive volatilisation of water (electrolyte), without substantial increases in either pressure or temperature within the bubble. Current continues to flow through the discharge as the bubble expands, and this growth (and the related increase in electrical resistance) is thought to be responsible for the current being cut off (soon after the point of maximum radius). A semiquantitative audit is presented of the transformations between different forms of energy that take place during the lifetime of a discharge.
\end{abstract}

Keywords: plasma electrolytic oxidation; electrical discharges; bubbles; high speed photography.

\section{Introduction}

The commercial significance of the plasma electrolytic oxidation (PEO) process continues to increase, but technical developments still depend largely on empirical experimentation. However, progress is being made on obtaining improved understanding of certain fundamental features particularly the main characteristics of the individual discharges. For example, their duration ( 30-300 $\mu \mathrm{s})$, spatial distribution (many localised "cascades" that persist for at least several tens of $\mathrm{ms})$, the "incubation" periods between individual discharges in a cascade $(\sim 0.1-1 \mathrm{~ms})$, the peak

\footnotetext{
*tel: 00441223 334332: e-mail: twc10@cam.ac.uk
} 
discharge current $(\sim 10-100 \mathrm{~mA})$, the discharge energy $(\sim 1-10 \mathrm{~mJ})$ and the diameter of the core discharge channels $(\sim 10-50 \mu \mathrm{m})$ are all now fairly well established [1-7], although they exhibit some dependence on substrate type, electrolyte composition and imposed electrical conditions. Correlations have also been established [3, 8, 9] between external conditions, discharge characteristics and features of resultant coatings, including the growth rate, and a start has been made $[7,10]$ on correlating the energetics of individual discharges with that of the process as a whole (with the ultimate aim of reducing the - often relatively high - rate of energy consumption).

There have also been extensive (time-averaged) spectrographic studies [1, 3, 11-16] of the discharges, aimed at establishing temperatures, charge densities and chemical compositions. There is now quite a lot of information available about these features, and the role of variables such as the frequency of the AC supply is now a little clearer. Some studies [8, 17-20] have revealed that, under certain conditions, the discharges can become very "diffuse" ("soft" regime), and it's also been shown recently [21-23] that discharges can in some cases occur during the cathodic part of the cycle, with both of these types of change affecting the growth characteristics and microstructure of the resultant coatings. However, it's not really been possible so far to explain properly why the conditions concerned lead to these changes in discharge characteristics, and in general it's evident that current understanding of the process as a whole is still far from complete.

A feature that has been noted, and (to a limited extent) investigated, by a few researchers [5-7, $14,24,25]$ is that gas bubbles (or "plasma bubbles") tend to form in the region where an individual discharge emerges into the electrolyte, apparently with a tendency to grow rapidly to a significant size (and then to shrink). Attempts have also been made [26] to explore how external introduction of (relatively large) gas bubbles can affect discharge formation. However, the phenomenon of bubble expansion from a discharge is clearly very different from that of external gas injection and indeed it also appears to be quite distinct from that of conventional electrolytic formation of gases (usually hydrogen and/or oxygen) at an electrode [27], which normally leads to escape of (relatively large) bubbles.

It has been speculated [5, 7, 25] that these discharge-linked bubbles, which apparently can rapidly oscillate in size, arise from pressure created as the hot plasma expands, and that this expansion leads to cooling, reduction in charge density, increase in electrical resistance, sharp curtailment of the discharge current and then collapse of the bubble. In fact, preliminary attempts have been made [5] to predict the bubble radius as a function of peak discharge current, although this model was based on several very crude exploratory assumptions, including neglect of the electrical resistance of the plasma, compared with that of the electrolyte (and also neglect of water volatilisation, viscous flow effects etc). The outcome was that the bubble radius was predicted to range up to about $30-40 \mu \mathrm{m}$, but there few systematic experimental data available at the time for comparison. Hamdan et al [25] did observe bubbles forming of up to several hundred microns in diameter (over periods of the order of $100 \mu \mathrm{s}$ ), although this was in heptane, rather than aqueous solution, and was under rather different conditions from those created during PEO. Vol'f et al [24] did observe "vapor-plasma" bubbles forming under PEO-like conditions, and reported that 
their lifetime was about $200 \mu \mathrm{s}$, but did not draw any conclusions about gas pressures or mechanisms of growth.

It seems likely that a full understanding of how and why this sequence of events involving the "plasma bubble" takes place will be very helpful for optimization of the process as a whole. The literature presently contains little or no solid experimental evidence in this area, mainly because there has hitherto been no synchronised monitoring of electrical and optical (imaging) information. The current paper presents synchronised data of this type, together with a preliminary investigation of how energy is redistributed during bubble expansion and contraction.

\section{Experimental Procedures}

\subsection{Sample Preparation}

PEO coatings were produced on Al-6082 substrates, which were in the form of $25.4 \mathrm{~mm}$ square section bars mounted in resin. A small piece of the bar was swaged to produce a wire of diameter $1 \mathrm{~mm}$. This wire was mounted in the resin, adjacent to the bar. Wire and bar were connected to a pair of PVC-insulated twisted wires extending out of the tank, where a $100 \Omega$ resistor connected the ends of the twisted wires. The set-up is depicted in Fig.1(a).

Coatings were prepared using a $100 \mathrm{~kW}$ Keronite $^{\mathrm{TM}}$ processing rig and an electrolyte consisting primarily of a dilute aqueous solution of potassium hydroxide and sodium silicate. The electrolyte was maintained at a temperature of approximately $20^{\circ} \mathrm{C}$ by re-circulation through a heat exchanger. The applied potential was nominally square-wave, with variable frequency, although the results presented here all relate to experiments carried out at $50 \mathrm{~Hz}$. A constant current condition was set, so as to achieve a current density of $31 \mathrm{~A} \mathrm{dm}^{-2}$. The applied voltage was therefore not pre-determined, but adjusted by the power supply to maintain the appropriate current. The voltage was in the range $500-600 \mathrm{~V}$ during the process. A coating of thickness about $5 \mu \mathrm{m}$ was created (by running the process for about 3 minutes) before the measurements were started.

\subsection{High Speed Video Capture}

The camera employed was a Photron FastcamSA 1.1, with the acquisition rate set at 125,000 frames per second ( $8 \mu$ s exposure time). The linear spatial resolution was $9 \mu \mathrm{m}-$ ie an area of $81 \mu^{2}$ per pixel. Typical images comprised $192 \times 144$ pixels, covering an area of $2.24 \mathrm{~mm}^{2}$, which was large enough to view the entire cross section of the small area (1 mm diameter) wire. Sample surfaces were viewed through a glass window in the electrolyte tank, as shown in Fig.1(a). The distance between lens and sample was $500 \mathrm{~mm}$. Image sequences were acquired after various PEO processing times, using different frequency waveforms. Due to the very high frame rates involved, it was necessary to illuminate the sample surface, in order to be able to see bubbles clearly. This was done using a high intensity flash (white light) source, which had a duration of approximately $10 \mathrm{~ms}$. The maximum total record time of the camera $(\sim 2 \mathrm{~s})$ was employed, since the flash was being triggered manually.

\subsection{Small Area Current Monitoring}

The small area electrical monitoring technique is described in detail elsewhere $[1,5,6]$. The concept behind the methodology is that discharge events on a small area take place in much the 
same way as those on "normal" (larger) samples, but with a strong probability that, when a discharge occurs, it will be the only one taking place at that time. By monitoring the current flowing through the small area sample, the current-time profile of individual discharges can thus be obtained.

The electrical circuit employed is represented in Fig.1(b). Electrical data were collected using a four-channel, 8-bit resolution PC oscilloscope (Pico Technology, Picoscope 6403), with a sample interval of $102.4 \mathrm{~ns}$. The Picoscope was operated using a desktop PC with a USB opto-isolator. This was necessary in order to avoid ground loops between the PC and the ground of the power supply. The voltage across the electrolyte tank was measured using a 100 potential divider and the bulk current was determined by measuring the voltage drop across a $1 \Omega$ resistor connected in series between the tank and ground. The end of the $1 \mathrm{~mm}$ diameter wire was used as the small area sample during the present investigation. The wire was connected to a bulk substrate through a $100 \Omega$ resistor, as shown in Fig.1(a), with the current determined from the voltage drop across the resistor, which was measured using an active differential probe (Pico Technology TA043) with $10 \times$ attenuation.

The high speed camera generated a TTL trigger signal ( $+5 \mathrm{~V}, 10 \mu$ s square wave) within $100 \mathrm{~ns}$ of the camera being activated. Since each frame corresponds to $8 \mu \mathrm{s}$, this $100 \mathrm{~ns}$ delay is considered negligible. The trigger signal was connected to one channel of the Picoscope, to initiate recording.

\subsection{Image Analysis}

The video sequences that were studied contained approximately 1,500 individual frames, covering the complete period of illumination by the flash $(\sim 10 \mathrm{~ms})$. For a particular discharge, the synchronised video sequence shows the expansion and subsequent collapse of a bubble. A sequence is presented here from a period when there were no other bubbles in the vicinity from previous discharges, which, if present, can perturb and interact with the growing bubble. Bubble radii were estimated from the size of best-fit ellipses superimposed on images, which were then converted to equivalent radii for a circle with the same area. (In general, most bubbles appeared approximately circular, indicating a hemi-spherical shape.)

\section{Synchronised Current and Video Monitoring}

\subsection{Electrical Characteristics of the Discharges}

A typical current-time relationship after initiation is shown in Fig.2. It can be seen that, after a transient period (lasting $\sim 1 \mathrm{~ms}$ ), current pulses start to form, representing individual discharges. As was observed from light emissions [7] under similar conditions (on a bulk sample), discharge durations were around 20-100 $\mu$ s (depending on coating thickness and current density) and periods between them were of the order of several hundred $\mu$ s (all occurring in cascades at particular locations). The discharge indicated in the figure is the one selected for synchronous study of video images.

Fig. 3 shows a higher resolution view of the current-time relationship for the selected discharge, together with the corresponding voltage-time relationship. In addition to allowing the duration of 
the pulse $(\sim 120 \mu \mathrm{s})$ to be accurately evaluated, this plot reveals that there are some spikes in the current profile. However, it's also clear from this figure that they arise from regular high frequency $(\sim 50 \mathrm{kHz})$ fluctuations in the applied voltage, which are present throughout and are unaffected by the presence or absence of current flow. They probably arise from some sort of source modulation or interference effect, and they are clearly unrelated to any phenomena associated with the individual discharges.

\subsection{Images of the Selected Discharge and Dynamics of Bubble Growth}

Fig.4 shows a selection from the sequence of video images associated with the discharge indicated in Fig.2. These 12 images, each with an exposure period of $8 \mu \mathrm{s}$, cover the period indicated in Fig. 3 as running from about $304.10 \mathrm{~ms}$ to $304.28 \mathrm{~ms}$ (ie about $180 \mu \mathrm{s}$ ) - every second image in the sequence is shown in this figure, so that the interval between them is $16 \mu \mathrm{s}$. It can be seen in this figure that the bubble expands more or less symmetrically from the bright spot representing the point where the discharge reaches the surface of the coating. It also contracts symmetrically. This bright spot remains visible up until the $304.216 \mathrm{~ms}$ image (arrowed), after which it disappears - there is still some light coming from the bubble area after that, although this appears to be due to reflections from the illuminating light source. This observation is consistent with the current-time plot in Fig.3, where it can be seen that the current is largely cut off at about $304.230 \mathrm{~ms}$.

It's certainly clear that electric current does continue to flow well after the bubble has started to form and grow. It's not so clear, however, what path this current is following during this phase. It may be going (diffusely) through the gaseous phase within the bubble, although there appears to be little or no (visible) plasma-like emission from this region (apart from the central spot, arrowed in one of the images, representing the discharge channel within the coating). There is also no evidence of a localised arc reaching the surface of the bubble. (It should perhaps be noted at this point that, while it would be unjustifiable to draw detailed general conclusions from study of a single discharge, data and images from many others in this investigation have been examined and the one under scrutiny here appears to be typical.) Alternatively, the current may be reaching the bulk of the electrolyte at least partly via other routes - for example, through liquid flow or ionic diffusion within electrolyte contained in the pores within the coating surrounding the discharge channel. On the other hand, it seems very likely that it is an increase in the electrical resistance of the growing bubble that is somehow responsible for cutting off the current. This issue needs further (experimental and theoretical) study.

It can also be seen that, once the current has stopped flowing, the bubble no longer contains a bright central spot. Furthermore, while the bright spot appears to have a radius of about $30 \mu \mathrm{m}$ (broadly consistent with the apparent size of discharges monitored previously solely via light emission), the bubble grows to a maximum radius of about $500 \mu \mathrm{m}$. This is apparent in Fig.5, which shows measured values of the bubble radius from all 22 of the video images covering this period, together with the current-time plot. A further feature is also apparent in this plot. The bubble radius actually starts to reduce (at $t=304.21 \mathrm{~ms}$ ) slightly before the current has stopped flowing (at $t=304.23 \mathrm{~ms}$ ), although the shrinkage rate does accelerate considerably after the current has been cut off. It seems likely that there is a ongoing balance between (electrical) 
injection of energy and the various (energy-absorbing) phenomena associated with expansion of the bubble; it's certainly possible that this balance could lead to the bubble starting to shrink while some injection of energy is still taking place.

\subsection{Relationship between Bubble Growth Rate and Gas Pressure}

Expansion of a (hemi-)spherical gas bubble in a liquid, driven by an internal over-pressure, $\Delta P$, is governed by the well-known Rayleigh-Plesset equation, which may be written

$$
\frac{\Delta P}{\rho_{\mathrm{L}}}=R\left(\frac{\mathrm{d}^{2} R}{\mathrm{~d} t^{2}}\right)+\frac{3}{2}\left(\frac{\mathrm{d} R}{\mathrm{~d} t}\right)^{2}+\frac{4 \mu_{\mathrm{L}}}{R}\left(\frac{\mathrm{d} R}{\mathrm{~d} t}\right)+\frac{2 \gamma}{\rho_{\mathrm{L}} R}
$$

where $R$ is the bubble radius, $\rho_{\mathrm{L}}$ is the density of the liquid $\left(\sim 1000 \mathrm{~kg} \mathrm{~m}^{-3}\right), \mu_{\mathrm{L}}$ is its kinematic viscosity $\left(\sim 10^{-6} \mathrm{~m}^{2} \mathrm{~s}^{-1}\right)$ and $\gamma$ is the energy of the gas-liquid interface $\left(\sim 0.05 \mathrm{~J} \mathrm{~m}^{-2}\right)$. For the present case, the right hand side is largely dominated by the second term. Applying the equation over the initial period of bubble growth, when the velocity of the interface is constant at about $10 \mathrm{~m} \mathrm{~s}^{-1}$ (see Fig.5), leads to a value for $\Delta P$ of about 1.5 bar. This pressure clearly drops off as the radius approaches its maximum, presumably because the rate of expansion of the bubble volume is no longer matched by the rate of vaporisation of water, but the main point to note is that the pressure never becomes large. This over-pressure value ( $\sim$ bar $)$ is consistent with the fact that the rate of radius contraction after the water vapour has started to condense is similar to the rate of expansion (ie the plot in Fig.5 is approximately symmetrical), with the shrinkage presumably driven by an external over-pressure of about 1 bar.

It may, however, be noted that the pressure at the point when the bubble first starts to form could be appreciably higher than the regime indicated above. The work of Hamdan et al [25] suggested that, immediately after ignition (within $\sim 100 \mathrm{~ns}$ ), the pressure may be tens or hundreds of atmospheres, but it falls off very quickly as the bubble expands. The temporal resolution of the present work is insufficient to draw any conclusions about effects occurring in these very early stages.

\section{Energetics of Individual Discharges}

\subsection{Energy associated with Coating Production and Heating}

In an earlier publication [7], a typical volume of substrate that is oxidised during one discharge was estimated to be $\sim 10 \mu \mathrm{m}^{3}$, corresponding to a mass of about $0.03 \mathrm{ng}$. This quantity of material must be melted and vaporised, and then raised to the temperature of the plasma. Estimates of the plasma temperature within a discharge vary considerably, but are no higher than about 10,000 K. The energy required, $Q$, to vaporise this mass, $m$, of material, and raise its temperature to $10,000 \mathrm{~K}$, can be calculated from the following equation

$$
Q=m C_{\mathrm{p}} \Delta T+m \Delta H_{\text {melt }}+m \Delta H_{\text {vap }}
$$

where the specific heat, $C_{\mathrm{p}}$, is taken to have a value of $\sim 1 \mathrm{~J} \mathrm{~g}^{-1} \mathrm{~K}^{-1}$ for the substrate material and $\Delta H_{\text {melt }}$ and $\Delta H_{\text {vap }}$ (latent heats of melting and vaporisation) have values of $400 \mathrm{~J} \mathrm{~g}^{-1}$ and $10,500 \mathrm{~J} \mathrm{~g}^{-1}$ respectively. Vaporising this quantity of material requires just $0.6 \mu \mathrm{J}$, which is an insignificant fraction of the total energy input $(\sim 1 \mathrm{~mJ})$. 
It should perhaps be noted at this point that it's not really clear whether the plasma formed during PEO can be considered to be in local thermal equilibrium (ie whether the effective temperatures of electrons and other species are similar). Most spectroscopic measurements suggest that it's probably not, at least in the early stages, and some estimates of the true effective temperature of the bulk of the material present are considerably lower (below 5,000 K). However, it's clear that the outcome of this calculation (ie that the energy required to raise the quantity of material present in the plasma to a very high temperature is negligible compared to the electrical energy injected) would be the same for any plausible temperature that could be used.

Melting of existing coating material surrounding the discharge also turns out to require relatively little energy, even though the quantity of alumina involved in this is considerably larger than the amount actually formed during each discharge. Taking the region of (alumina) coating to be melted as a cylinder of radius $10 \mu \mathrm{m}$ and thickness $5 \mu \mathrm{m}$, the mass concerned is $\sim 6 \mathrm{ng}$. Assuming that the molten coating remains at the melting temperature of alumina $(\sim 2,300 \mathrm{~K})$, and taking its latent heat of melting, $\Delta H_{\text {melt, }}$ to be $1,150 \mathrm{~J} \mathrm{~g}^{-1}$, the energy required is obtained from

$$
Q=m C_{\mathrm{p}} \Delta T+m \Delta H_{\text {melt }}
$$

giving a value of $\sim 20 \mu \mathrm{J}$. This is greater than that required to vaporise the metal being oxidised, but is still much less than the total electrical energy being injected. This is perhaps encouraging in terms of suggesting that the irreducible minimum amount of energy associated with a process of this type may be much smaller than the quantity that is typically consumed.

\subsection{Energy of Plasma Formation and Sustainment}

The energy required to create and sustain the plasma is more difficult to calculate, and the following analysis contains some crude approximations. Creation of the plasma requires ionisation of atoms. The electron density within the plasma has been estimated experimentally (via spectroscopic measurements) to be between $10^{12}$ and $10^{14} \mathrm{~mm}^{-3}$. The ionisation energy (H and $\mathrm{O}$ atoms) is of the order of $2 \times 10^{-18} \mathrm{~J}_{\text {atom }}{ }^{-1}$. An upper bound can be obtained by assuming that the entire gas bubble $\left(r_{\mathrm{B}} \sim 500 \mu \mathrm{m}\right)$ has an electron density of $10^{14} \mathrm{~mm}^{-3}$. It follows that the energy required to ionise sufficient atoms would be $\sim 50 \mu \mathrm{J}$. This is again relatively small, although perhaps not entirely insignificant, in the context of an injected energy of around $1 \mathrm{~mJ}$. Of course, this takes no account of the energy needed to sustain the plasma, which is more difficult to estimate. The maximum contribution from this source is likely to be about twice that for ignition. In any event, even making upper limit assumptions, the value is still small compared with the total injected energy.

\subsection{Energy of Vaporisation of Water}

The main reason why the above contributions to the energy audit are all rather insignificant is simply that the quantity of material involved in each case must be very small. However, this is not necessarily true for vaporisation of water (electrolyte). An estimate of interest is the mass, $m$, of water that could be vaporised by the total amount of energy injected by the discharge, $E$. The specific heat of water, $C_{\mathrm{p}}$, is $4.2 \mathrm{~J} \mathrm{~g}^{-1} \mathrm{~K}^{-1}$ and its latent heat of vaporisation, $\Delta H_{\mathrm{vap}}$ is $2,260 \mathrm{~J} \mathrm{~g}^{-1}$. For water initially at $298 \mathrm{~K}$ to be heated to $373 \mathrm{~K}$ and then vaporised (and remain at this temperature), the mass, $m$, for which this can be done is obtainable from 


$$
E=m C_{\mathrm{p}} \Delta T+m \Delta H_{\text {vap }}
$$

Assuming ideal gas behaviour, with atmospheric pressure and a hemi-spherical geometry, the following relationships apply between gas volume, $V$, gas pressure, $P$, absolute temperature, $T$, gas constant, $R$, number of moles of gas, $n$, and bubble radius, $r_{\mathrm{B}}$

$$
\begin{aligned}
& V=\frac{n R T}{P} \\
& r_{B}=\sqrt[3]{\frac{3 V}{2 \pi}}
\end{aligned}
$$

A value for the discharge energy, $E$, of $\sim 1.5 \mathrm{~mJ}$ (obtained by numerical integration of the product of voltage and current) then leads to a mass, $m$, of $580 \mathrm{ng}$, corresponding to $n \sim 3.2 \times 10^{-8}$ mol. This mass of water, in the liquid state, would occupy a hemi-sphere of radius $\sim 65 \mu \mathrm{m}$ and would, as a gas at atmospheric pressure, generate a bubble of radius $\sim 780 \mu \mathrm{m}$. Heat transfer to the coating, substrate and surrounding electrolyte have been neglected here, but would all reduce the energy available to vaporise the electrolyte.

In general, however, it seems clear that the quantity of water that would need to be vaporised in order to absorb at least most of the injected energy is quite plausible in terms of the volume of the hemi-sphere (of water vapour) that would be created (assuming that the pressure in it remains close to atmospheric - which the calculation of $\$ 3.3$ suggests that it will). Certainly, in the absence of water vaporisation, it's difficult to see how the dramatic expansion in bubble volume (relative to the volume of the discharge channel in the coating) could possibly occur without the pressure in it dropping well below atmospheric, in which case it would not be able to expand. It's easy to show that thermal expansion of this small quantity of material cannot account for the observed bubble growth - it would require heating to a completely unrealistic temperature, and of course it's already very hot when inside the discharge channel. It's unclear at this point whether there is significant ionisation of this (large quantity of) vaporised water, but on balance it seems unlikely.

\subsection{Electrical Energy Dissipation Directly in the Electrolyte}

It's fairly clear that, whatever forms the injected energy may transiently take after injection, most of it will end up as a raised temperature of the electrolyte, which in turn will have to be carried away by some form of heat exchanger if the process is to continue for any length of time. For example, if much of it does indeed get transformed from electrical/plasma energy to being stored in the form of a relatively large quantity of water vapour, this will subsequently condense, releasing this heat into the bulk of the electrolyte. However, it is possible that some of the electrical energy could be transferred directly into the electrolyte. The resistance, $R$, of the electrolyte through which the current is passing can be estimated using [5]

$$
\begin{gathered}
R=\frac{\rho}{2 \pi r_{\mathrm{B}}} \\
\Delta U=\frac{I \rho}{2 \pi r_{\mathrm{B}}}
\end{gathered}
$$


where $\rho$ is the resistivity of the electrolyte. The conductivity of the electrolyte was $5.1 \mathrm{mS} \mathrm{cm}^{-1}$, corresponding to a resistivity of around $2 \Omega \mathrm{m}$ (although stirring of the electrolyte may increase this). At the maximum bubble radius $(\sim 500 \mu \mathrm{m})$, the current, $I$, flowing through the discharge was $\sim 30 \mathrm{~mA}$, giving a voltage drop across the electrolyte, $\Delta U$, of $\sim 20 \mathrm{~V}$. Since the total voltage drop was of the order of $500 \mathrm{~V}$, the assumption that all the electrical energy is dissipated in the discharge site, or at least in very close proximity to it, appears to be valid.

\subsection{Chemical Energy of Oxidation}

Of course, the underlying chemical reaction is simply that of aluminium being oxidised to alumina. This contribution to the energy audit differs from all of the others, in that it is negative in sign - ie this energy will be released, rather than absorbed. Its magnitude is straightforward to estimate. The quantity of $\mathrm{Al}$ being transformed per discharge is about $0.03 \mathrm{ng}$ (see $\$ 4.1$ ), leading to the formation of about $0.057 \mathrm{ng}$ of $\mathrm{Al}_{2} \mathrm{O}_{3}$. The free energy of the reaction is about $1,500 \mathrm{~kJ}$ per mole (ie per $102 \mathrm{~g}$ ) of alumina formed. The energy released per discharge is thus about $1 \mu \mathrm{J}$. This is much smaller than all of the other contributions, reflecting the very low efficiency of the PEO process from an energetic point of view.

\subsection{Summary of Energy Audit Conclusions}

The above estimates of the quantities of energy, in different forms, associated with a single discharge are, of course, relatively crude, although they are certainly informed by the newlyacquired experimental information that is reported here. A semi-quantitative attempt to present this information graphically is shown in Fig.6 (omitting the chemical oxidation energy, which is negligible compared to the other contributions). This depicts the relative significance of the heating and phase changes experienced by the different constituents present. Evidently, assuming that this summary is reasonably accurate, most of the injected energy is (transiently) absorbed in the form of vaporisation of water, after which it will inevitably end up as rather low grade (difficult to recover) energy in the form of a large mass of (moderately) heated electrolyte.

There does not appear to be any immediately obvious way of reducing this energy wastage, although improved understanding of details such as those presented here is certainly of potential value in considering various measures. The basic problem appears to be that exposing the substrate to oxidising agents via these highly energetic discharges is very inefficient, at least under the conditions conventionally employed. Suppressing bubble growth (for example, by pressurising the liquid) would not necessarily reduce the energy wastage, although measures such as this might be worth exploring. The most promising approach is probably to somehow reduce the energy associated with each discharge (while retaining a similar rate of transformation of substrate), or to promote more transformation per discharge (while retaining a similar discharge energy). Further study of the details of the energetics of the process is clearly likely to be helpful in pursuing such aims.

\section{Conclusions}

The information presented (from synchronised current monitoring and high speed video imaging of individual discharges formed during PEO processing at $50 \mathrm{~Hz}$ AC) allows the following conclusions to be drawn: 
(a) The ignition of a discharge, and creation of a plasma channel through the thickness of the coating, is quickly followed by formation and growth of a (hemi-spherical) gas bubble, centred on the plasma channel (which probably has a radius ranging up to a few tens of $\mu \mathrm{m}$, depending on coating thickness).

(b) This bubble grows rapidly, typically reaching a radius of about $500 \mu \mathrm{m}$ during the lifetime of the discharge, which is of the order of $100 \mu \mathrm{s}$. It seems likely that this bubble growth is responsible for a sharp increase in the electrical resistance of the current path, leading to the current shutting off. From the observed rate of growth of the bubble, it's concluded that the peak over-pressure within it is relatively low (of the order of 1 atmosphere).

(c) It is deduced that this bubble growth occurs as a consequence of the rapid volatilisation of a large quantity of water (electrolyte), which remains at relatively low temperature and pressure as bubble expansion occurs. Once the discharge current has been shut off, the bubble contracts rapidly as this water vapour condenses, releasing most of the energy of the discharge into the bulk of the electrolyte and raising its temperature.

(d) A semi-quantitative audit has been carried out concerning the injected (electrical) energy and its transformation between the forms represented by the existence of the plasma, the melting and volatilisation of substrate and coating, the vaporisation of water to create the bubble and the heating of the bulk electrolyte.

\section{Acknowledgements}

This work has been supported by EPSRC (grant number EP/I001174/1), by a Sims Scholarship (for SCT) in Cambridge University and by Keronite plc. The research also forms part of the activities of the COST TD 1208 Network. Thanks are due to Steve Hutchins and Suman Shrestha, of Keronite, for many helpful discussions. The technical assistance of Fréderic Brochard (Nancy) and Kevin Roberts (Cambridge) is also gratefully acknowledged.

In compliance with EPSRC requirements, raw data in the form of selected video and discharge current files are available at www.ccg.msm.cam.ac.uk/publications/resources, and are also accessible via the University repository at http://www.data.cam.ac.uk/repository. 


\section{References}

[1] Dunleavy, CS, IO Golosnoy, JA Curran and TW Clyne, Characterisation of Discharge Events During Plasma Electrolytic Oxidation. Surf. Coat. Techn., 2009. 203: p.3410-3419.

[2] Kasalica, B, M Petkovic, I Belca, S Stojadinovic and L Zekovic, Electronic Transitions During Plasma Electrolytic Oxidation of Aluminum. Surf. Coat. Techn., 2009. 203: p.3000-3004.

[3] Martin, J, A Melhem, I Shchedrina, T Duchanoy, A Nomine, G Henrion, T Czerwiec and T Belmonte, Effects of Electrical Parameters on Plasma Electrolytic Oxidation of Aluminium. Surf. Coat. Techn., 2013. 221: p.70-76.

[4] Hussein, RO, X Nie and DO Northwood, A Spectroscopic and Microstructural Study of Oxide Coatings Produced on a Ti-6al-4v Alloy by Plasma Electrolytic Oxidation. Materials Chemistry and Physics, 2012. 134(1): p.484-492.

[5] Dunleavy, CS, JA Curran and TW Clyne, Self-Similar Scaling of Discharge Events through Peo Coatings on Aluminium. Surf. Coat. Techn., 2011. 206: p.1051-1061.

[6] Dunleavy, CS, JA Curran and TW Clyne, Time Dependent Statistics of Plasma Discharge Parameters During Bulk Ac Plasma Electrolytic Oxidation of Aluminium. Applied Surface Science, 2013. 268(0): p.397-409.

[7] Nomine, A, SC Troughton, AV Nomine, G Henrion and TW Clyne, High Speed Video Evidence for Localised Discharge Cascades During Plasma Electrolytic Oxidation. Surf. Coat. Techn., 2015. 269: p.125-130.

[8] Melhem, A, G Henrion, T Czerwiec, JL Briancon, T Duchanoy, F Brochard and T Belmonte, Changes Induced by Process Parameters in Oxide Layers Grown by the Peo Process on Al Alloys. Surf. Coat. Techn., 2011. 205: p.S133-S136.

[9] Habazaki, H, S Tsunekawa, E Tsuji and T Nakayama, Formation and Characterization of Wear-Resistant Peo Coatings Formed on B-Titanium Alloy at Different Electrolyte Temperatures. Applied Surface Science, 2012. 259: p.711-718.

[10] Matykina, E, R Arrabal, A Pardo, M Mohedano, B Mingo, I Rodriguez and J Gonzalez, Energy-Efficient Peo Process of Aluminium Alloys. Materials Letters, 2014. 127: p.13-16.

[11] Mécuson, F, T Czerwiec, T Belmonte, L Dujardin, A Viola and G Henrion, Diagnostics of an Electrolytic Microarc Process for Aluminium Alloy Oxidation. Surf. Coat. Techn., 2005. 200: p.804-808.

[12] Wang, L, L Chen, Z Yan and W Fu, Optical Emission Spectroscopy Studies of Discharge Mechanism and Plasma Characteristics During Plasma Electrolytic Oxidation of Magnesium in Different Electrolytes. Surf. Coat. Techn., 2010. 205: p.1651-1658.

[13] Hussein, RO, X Nie and DO Northwood, Influence of Process Parameters on Electrolytic Plasma Discharging Behaviour and Aluminum Oxide Coating Microstructure. Surf. Coat. Techn., 2010. 205: p.1659-1667.

[14] Wang, L, W Fu and L Chen, Evolution of Active Species and Discharge Sparks in Na2sio3 Electrolyte During Peo Process. Journal of Alloys and Compounds, 2011. 509(28): p.7652-7656.

[15] Jovović, J, S Stojadinović, NM Šišović and N Konjević, Spectroscopic Characterization of Plasma During Electrolytic Oxidation (Peo) of Aluminium. Surf. Coat. Techn., 2011. 206: p.24-28.

[16] Stojadinović, S, R Vasilić, M Petković, B Kasalica, I Belča, A Žekić and L Zeković, Characterization of the Plasma Electrolytic Oxidation of Titanium in Sodium Metasilicate. Applied Surface Science, 2013. 265(0): p.226-233.

[17] Jaspard-Mécuson, F, T Czerwiec, G Henrion, T Belmonte, L Dujardin, A Viola and J Beauvir, Tailored Aluminium Oxide Layers by Bipolar Current Adjustment in the Plasma Electrolytic Oxidation (Peo) Process. Surf. Coat. Techn., 2007. 201: p.8677-8682

[18] Arrabal, R, E Matykina, T Hashimoto, P Skeldon and GE Thompson, Characterization of Ac Peo Coatings on Magnesium Alloys. Surf. Coat. Techn., 2009. 203: p.2207-2220.

[19] Matykina, E, R Arrabal, P Skeldon, GE Thompson and P Belenguer, Ac Peo of Aluminium with Porous Alumina Precursor Films. Surf. Coat. Techn., 2010. 205: p.1668-1678.

[20] Martin, J, P Leone, A Nomine, D Veys-Renaux, G Henrion and T Belmonte, Influence of Electrolyte Ageing on the Plasma Electrolytic Oxidation of Aluminium. Surf. Coat. Techn., 2015. 269: p.36-46. 
[21] Nomine, A, J Martin, C Noel, G Henrion, T Belmonte, IV Bardin, VL Kovalev and AG Rakoch, The Evidence of Cathodic Micro-Discharges During Plasma Electrolytic Oxidation Process. Appl. Phys. Lett., 2014. 104(8): p.Art. 081603.

[22] Nomine, A, J Martin, G Henrion and T Belmonte, Effect of Cathodic Micro-Discharges on Oxide Growth During Plasma Electrolytic Oxidation (PEO). Surf. Coat. Techn., 2015. 269: p.131-137.

[23] Rakoch, AG, AA Gladkova, Z Linn and DM Strekalina, The Evidence of Cathodic MicroDischarges During Plasma Electrolytic Oxidation of Light Metallic Alloys and MicroDischarge Intensity Depending on Ph of the Electrolyte. Surf. Coat. Techn., 2015. 269: p.138-144.

[24] Vol'f, E, A Sizikov and L Bugaenko, Determination of the Mean Lifetime of Vapor-Plasma Bubbles for a Microdischarge on Aluminum Valve Anode in Aqueous Electrolyte Solution. High Energy Chemistry, 1998. 32: p.407-410.

[25] Hamdan, A, C Noel, F Kosior, G Henrion and T Belmonte, Dynamics of Bubbles Created by Plasma in Heptane for Micro-Gap Conditions. Journal of the Acoustical Society of America, 2013. 134(2): p.991-1000.

[26] Lee, J, Y Kim and W Chung, Effect of Ar Bubbling During Plasma Electrolytic Oxidation of Az31b Magnesium Alloy in Silicate Electrolyte. Applied Surface Science, 2012. 259: p.454-459.

[27] Guo, H, M An, S Xu and H Huo, Formation of Oxygen Bubbles and Its Influence on Current Efficiency in Micro-Arc Oxidation Process of Az91d Magnesium Alloy. Thin Solid Films, 2005. 485: p.53-58. 


\section{Figure Captions}

Fig.1 Schematic representation of the experimental arrangement, showing (a) a depiction of the set-up and (b) a circuit diagram.

Fig.2 Section of the current-time relationship for the small area sample.

Fig.3 Synchronised current-time and voltage-time relationships for the small area sample, focussed on the period of the individual discharge indicated in Fig.2. The vertical dotted lines indicate the times at which the bubble associated with this discharge first appeared and finally disappeared.

Fig.4 Sequence of video images covering the time period indicated in Fig.3.

Fig.5 Synchronised current-time and bubble radius-time relationships for the small area sample, focussed on the period of the individual discharge indicated in Fig.2.

Fig.6 Semi-quantitative plot showing how the total (electrically-injected) energy changes during formation of the individual discharge under consideration here, and how it is converted between different forms during and immediately after the discharge period. 


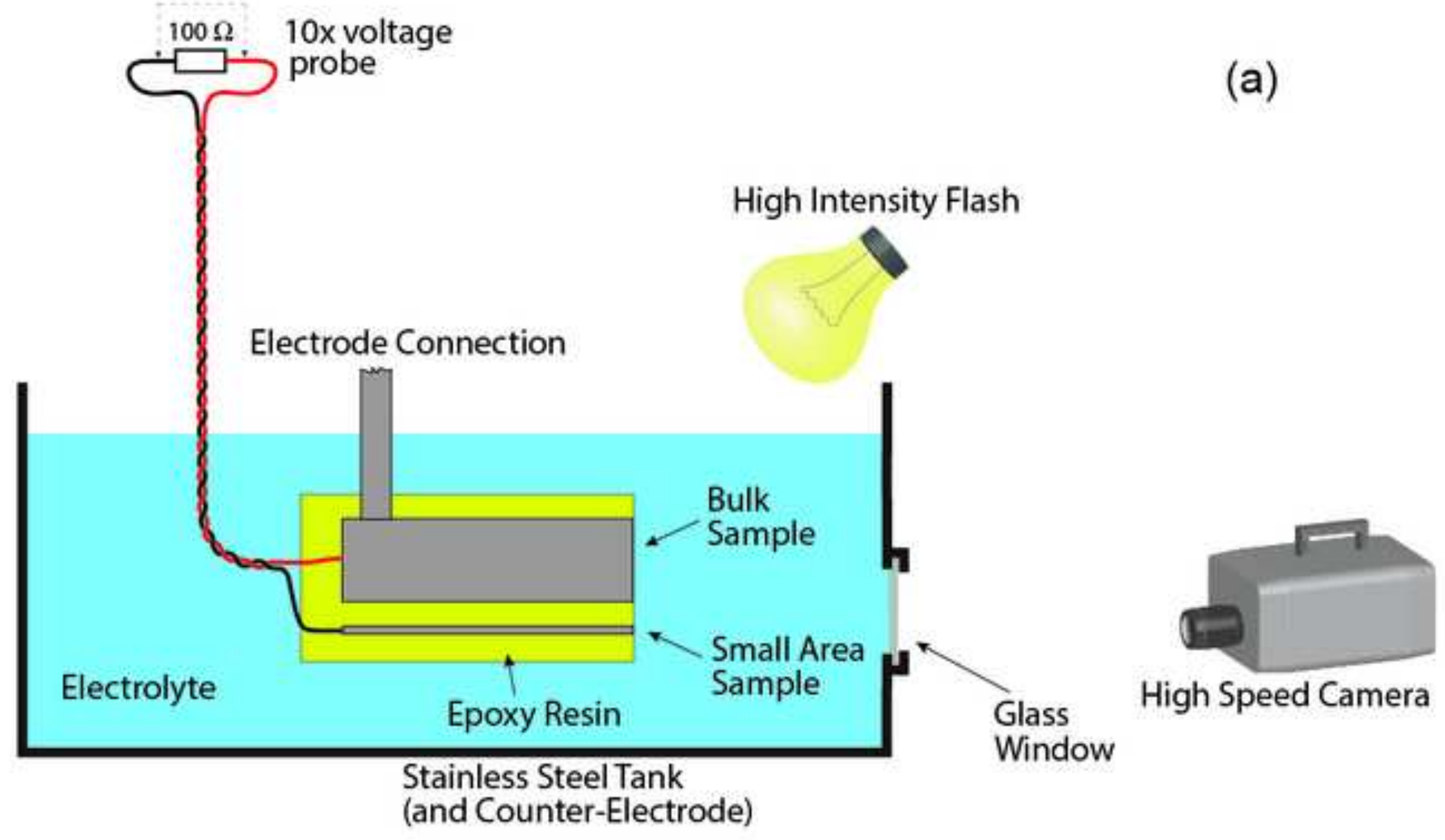




\section{0 kW Power Supply}

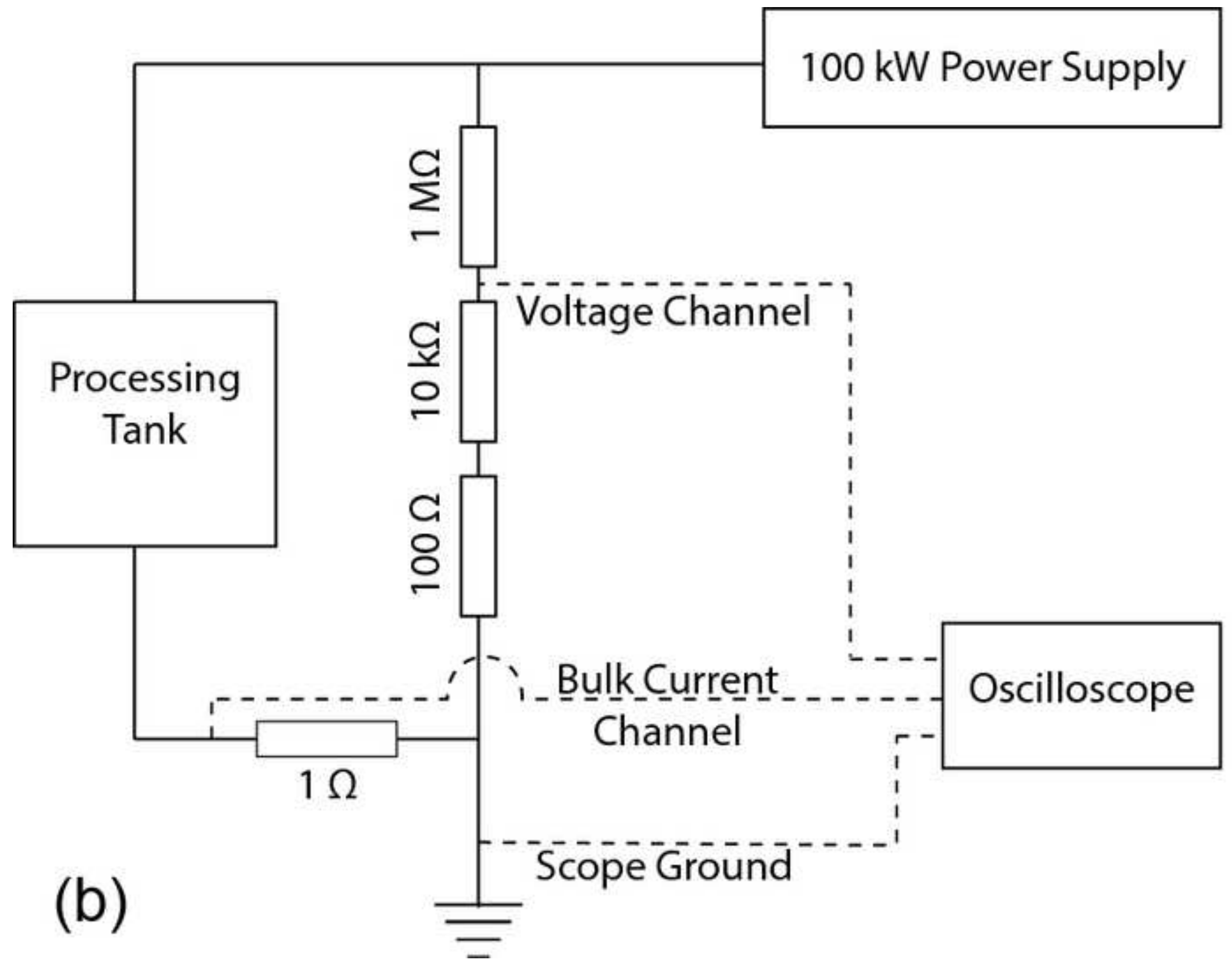




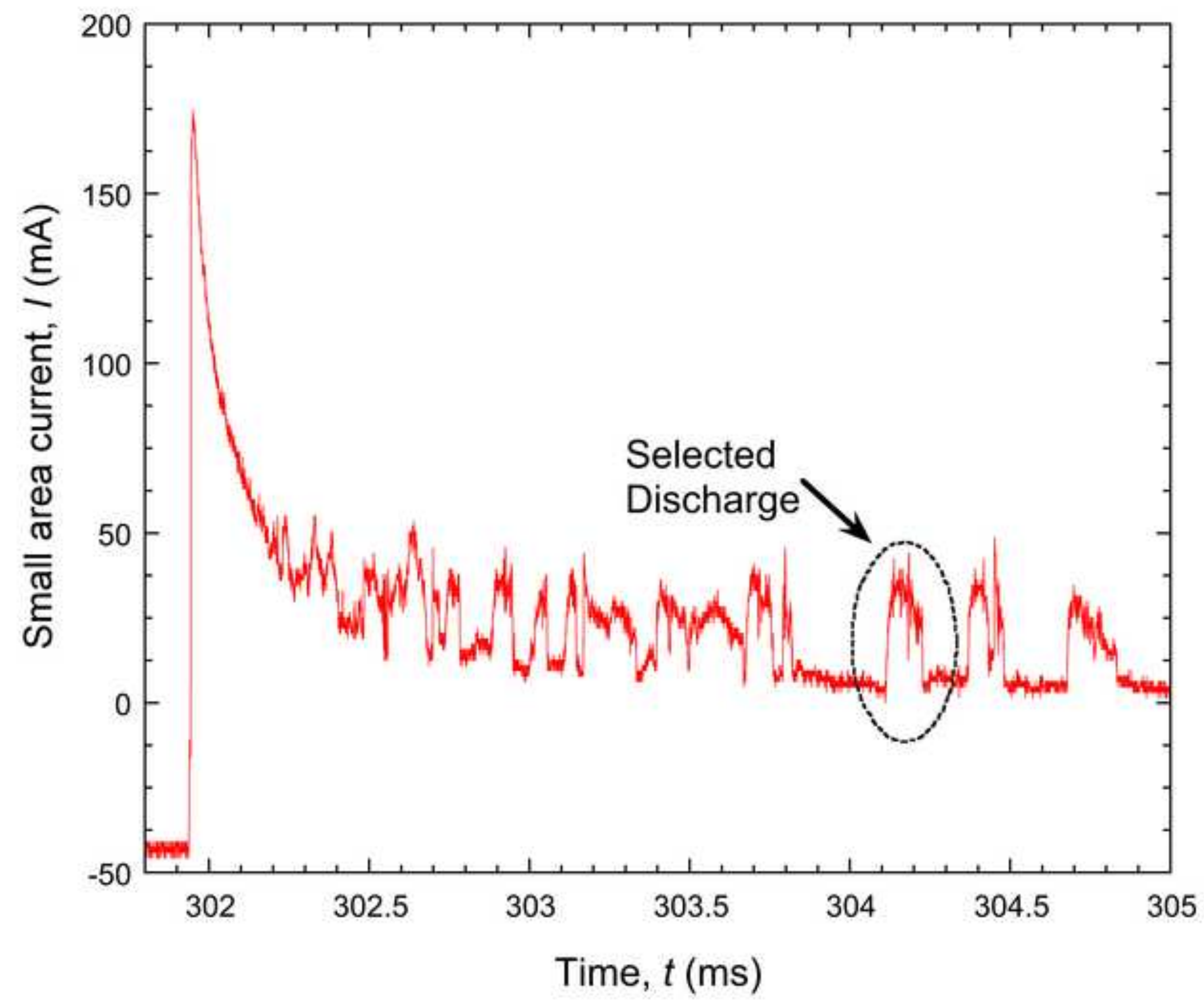




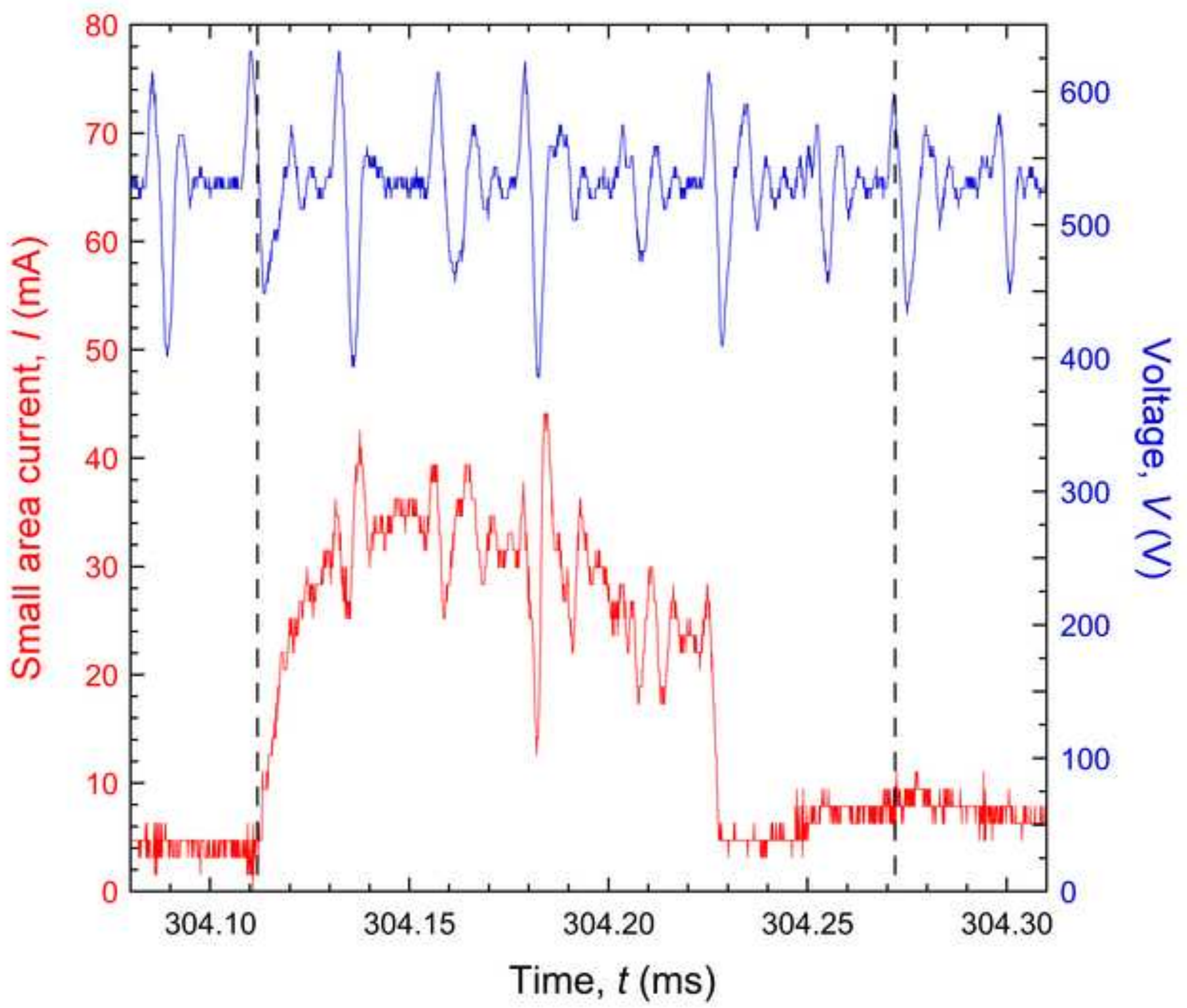




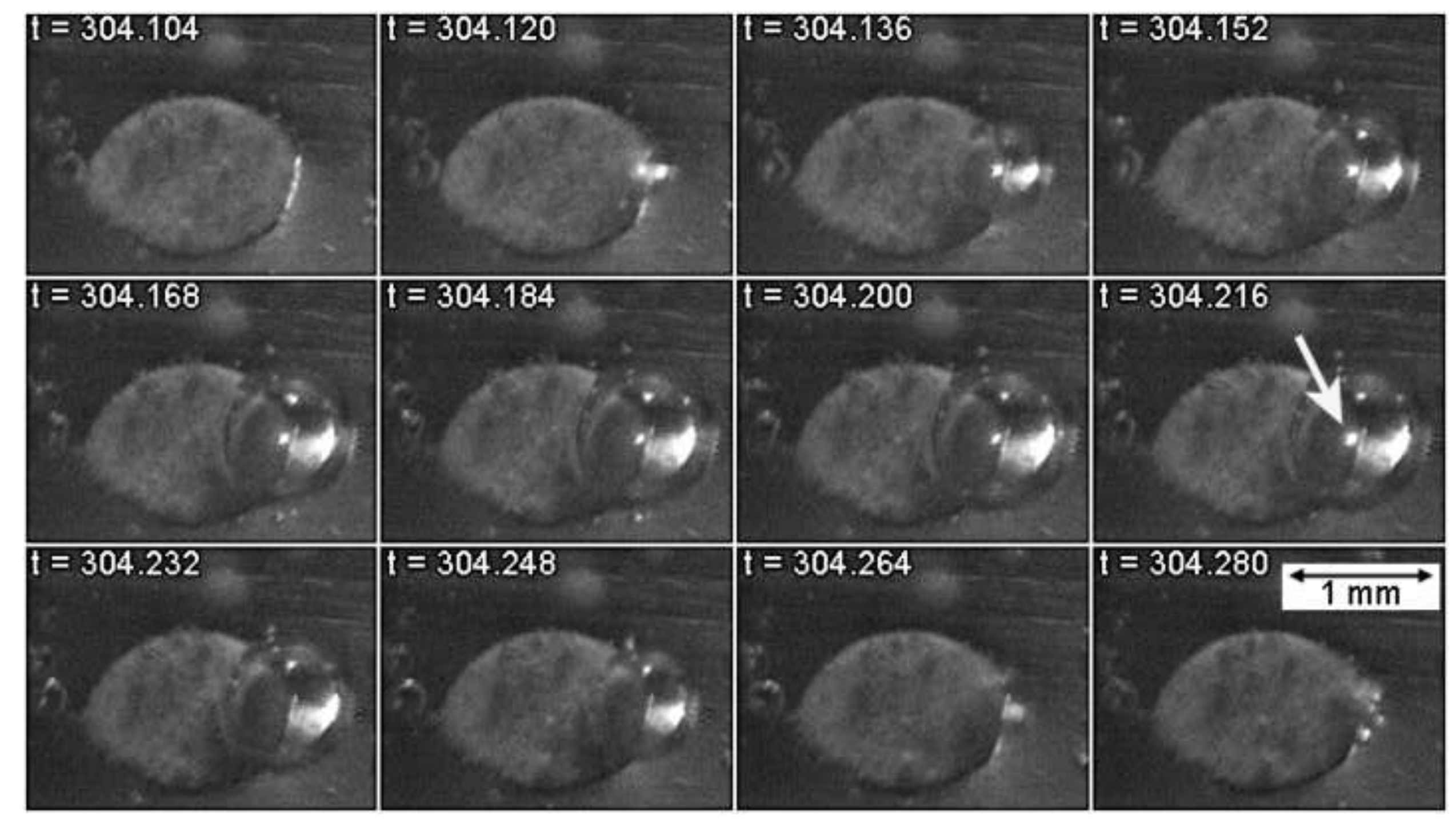




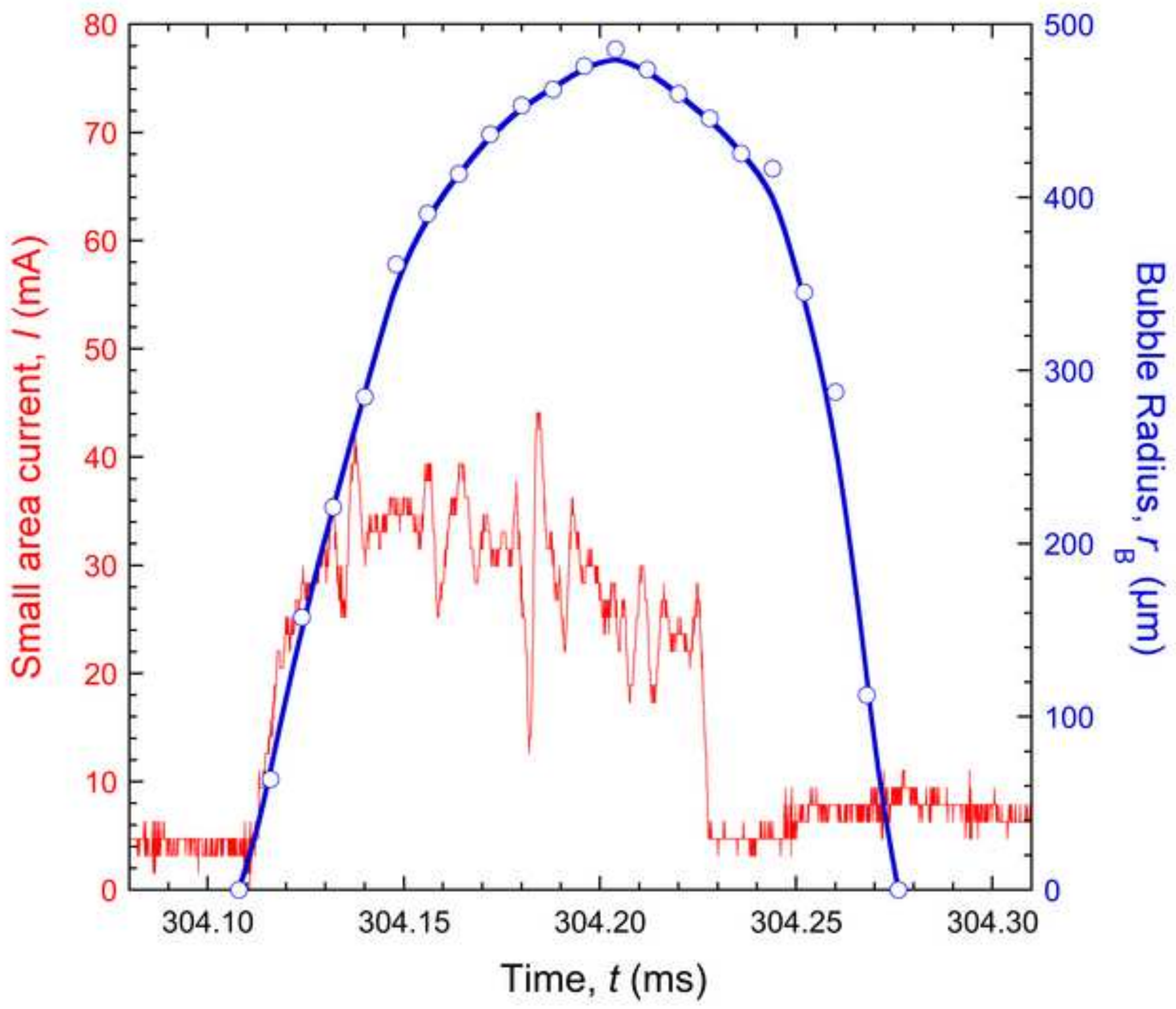




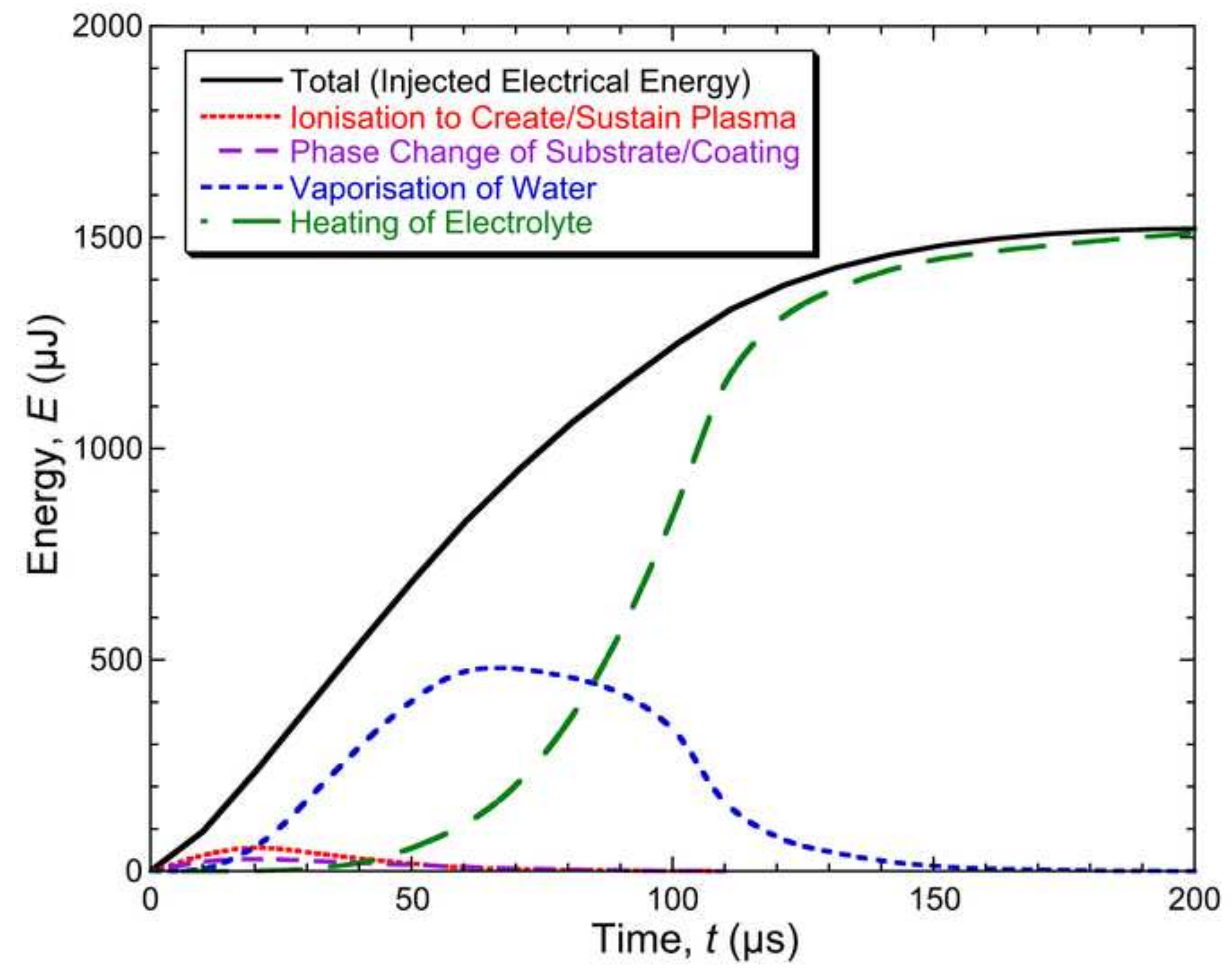

